\title{
Microwave photonics processing controlling the speed of light in semiconductor waveguides
}

[invited]

Xue, Weiqi; Chen, Yaohui; Sales, Salvador; Blaaberg, Søren; Mørk, Jesper; Capmany, Jose

Published in:

11th International Conference on Transparent Optical Networks, 2009. ICTON '09

Link to article, DOI:

10.1109/ICTON.2009.5185046

Publication date:

2009

Document Version

Publisher's PDF, also known as Version of record

Link back to DTU Orbit

Citation (APA):

Xue, W., Chen, Y., Sales, S., Blaaberg, S., Mørk, J., \& Capmany, J. (2009). Microwave photonics processing controlling the speed of light in semiconductor waveguides: [invited]. In 11th International Conference on

Transparent Optical Networks, 2009. ICTON '09 (pp. 1-5). IEEE. https://doi.org/10.1109/ICTON.2009.5185046

\section{General rights}

Copyright and moral rights for the publications made accessible in the public portal are retained by the authors and/or other copyright owners and it is a condition of accessing publications that users recognise and abide by the legal requirements associated with these rights.

- Users may download and print one copy of any publication from the public portal for the purpose of private study or research.

- You may not further distribute the material or use it for any profit-making activity or commercial gain

- You may freely distribute the URL identifying the publication in the public portal 


\title{
Microwave Photonics Processing Controlling the Speed of Light in Semiconductor Waveguides
}

\author{
Weiqi Xue ${ }^{1}$, Yaohui Chen ${ }^{1}$, Salvador Sales ${ }^{2}$, Søren Blaaberg ${ }^{1}$, Jesper Mørk ${ }^{1}$ and José Capmany ${ }^{2}$ \\ 1: DTU Fotonik, Department of Photonics Engineering, Technical University of Denmark \\ Build. 343, DK-2800 Kgs. Lyngby, Denmark \\ 2: ITEAM; Institute of Telecom.s and Multimedia Application, Universidad Politécnica de Valencia \\ Camino de Vera s/n, 46020 Valencia, Spain \\ Tel: +34 963879735,Fax:+34 963877307,e-mail: ssales@dcom.upv.es
}

\begin{abstract}
We review the theory of slow and fast light effect in semiconductor waveguides and potential applications of these effects in microwave photonic systems as RF phase shifters. Recent applications as microwave photonic filters is presented. Also, in the presentation more applications like optoelectronic oscillators and arbitrary waveform generators will be described. Some work related to the noise and distortion will also be discussed.

Keywords: microwave photonics, slow/fast light, microwave phase shifter.
\end{abstract}

\section{INTRODUCTION}

MicroWave Photonics (MWP) is defined as the collection of techniques that employ the photonic devices operating at microwave frequencies. The idea behind is to use the advantages of photonic devices compared to microwave devices to process the information. Thus, in some parts of the microwave systems, some subsystems are implemented in the optical domain. The number of applications is huge [1-3]. One of the key components is the Photonic Microwave Phase Shifter (PMPS). The PMPS is a photonic component that has to shift till 360 degrees the microwave signal that it is carried onto the optical signal. It has to be wideband and smoothly tunable. A versatile, compact and flexible PMPS will increase dramatically the applications of the MWP techniques. Several technologies have been proposed for the PMPS since the early beginning of the MWP till the recent days: fiber optics [4], optical filter with complex phase-response [5], liquid crystals [6], chirped FBGs [7], electrooptic polymers [8] amongst others.

The recent demonstration of slow light propagation in ultra-cold atomic gasses [9] has been the beginning of a new technique to create phase shifts in the microwave signals in the optical domain. To implement the new technique in a cost-effective way it has been necessary to demonstrate the phase shifts at room temperature [10]. And finally, the important issue has been to create this phase shift in semiconductor waveguides at room temperature [11]. The main advantages of using semiconductor waveguides compared with other photonic techniques are based on the semiconductor properties: the high tuning speed, the continuous scan of the phase shift and amplitude variations, the small size, the capability of integration, the low insertion losses and the low electrical power consumption.

\section{PRINCIPLE OF OPERATION}

The coherent population oscillation $(\mathrm{CPO})_{2}$ mechanism has been used to describe the Slow/Fast Light (SFL) effect in semiconductor waveguides. CPO is described in Fig. 1a. A weak probe with an optical frequency slightly detuned from that of the strong control creates that the carrier population of the conduction and valence bands oscillate at the beating frequency determined by the frequency detuning $\Delta \omega$. This carrier oscillation changes the absorption or gain seen by the probe. Thus, a coherent spectral hole appears in the imaginary part of the susceptibility, as shown in Fig. 1b. In semiconductor waveguides, the coherent spectral hole is characterized by the carrier lifetime $\tau_{s}$. Based on the Kramers-Kronig relation, this dip leads to a change of the refractive index dispersion shown in Fig. 1c, which finally accounts for the SFL effects via the change in the frequency slope of the refractive index. To produce the SFL effect in the microwave signals, a CW laser is modulated by a RF signal $(\Omega)$. In the frequency-domain, the modulated optical signal is comprised of the strong carrier, $\omega_{0}$, and two weak sidebands, red-shifted sideband $\omega_{0}-\Omega$ and blue-shifted sideband $\omega_{0}+\Omega$. Due to the modulation of the intensity, the gain and refractive index are modulated in time creating two temporal gratings, modifying the susceptibilities seen by the probes. Therefore, after the propagation in the semiconductor waveguide, a time delay or advance of $\Delta t$ appears in the time domain. After the photodetection, this time delay or advance also corresponds to a RF phase shift of $\Delta t \Omega$ at the microwave frequency $\Omega$.

Based on the model in [12], for an amplitude modulated signal with double sidebands, as shown in Fig. 2, the modulation refractive index can be derived as follows,

$$
n_{\text {mod }}(z)=n+\omega_{0} \frac{n\left(\omega_{0}+\Omega\right)-n\left(\omega_{0}-\Omega\right)}{2 \Omega} \cong n_{g b}-c \tau_{s} \Gamma g_{0} \frac{P(z) / P_{\text {sat }}}{1+P(z) / P_{\text {sat }}} \frac{1}{\left(\Omega \tau_{s}\right)^{2}+\left(1+P(z) / P_{\text {sat }}\right)^{2}}
$$


Here $n_{g b}$ is the background group refractive index, $P$ is the optical power, $P_{\text {sat }}$ is the saturation power, $g_{0}$ is the small signal gain (for an EA, $g_{0}$ is negative), $\Gamma$ is the confinement factor, and $c$ is the light velocity in vacuum. The corresponding microwave phase shift of the intensity modulated envelope, which is the quantity of interest in microwave photonics, can be expressed,

$$
\Delta \varphi_{R F}=\Omega \Delta t=\frac{\Omega}{c} \int_{0}^{L} n_{\mathrm{mod}}(z) d z-\Omega \frac{L}{c} n_{g b}=-\Omega \tau_{s} \Gamma g_{0} \int_{0}^{L} \frac{P(z) / P_{\text {sat }}}{1+P(z) / P_{\text {sat }}} \frac{1}{\left(\Omega \tau_{s}\right)^{2}+\left(1+P(z) / P_{\text {sat }}\right)^{2}} d z
$$

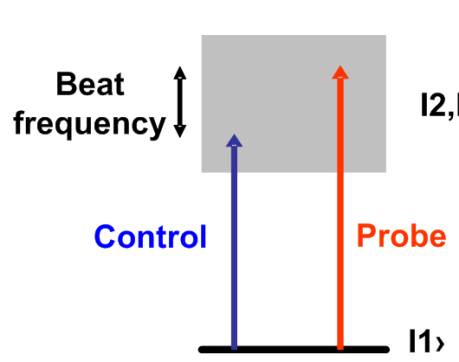

(a)

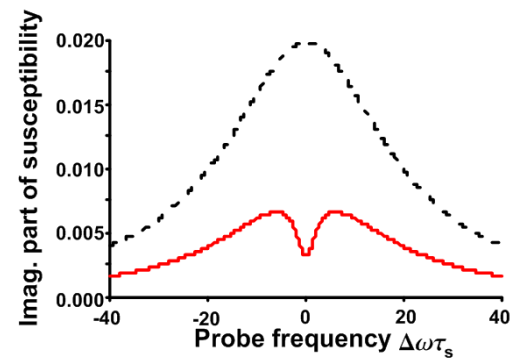

(b)

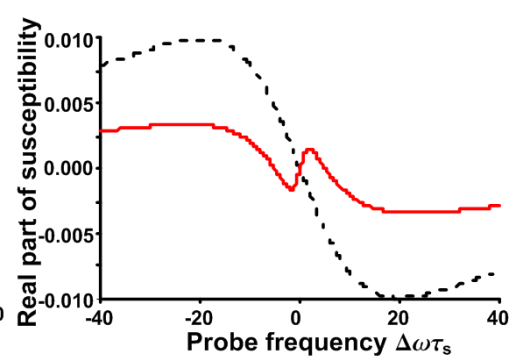

(c)

Fig. 1. (a) Level diagram in the presence of a strong control and weak detuned probe, and typical examples of the imaginary (b) and real (c) parts of the susceptibility experienced by the probe as a function of detuning frequency for CPO effects. The dashed lines show the susceptibility without the control.

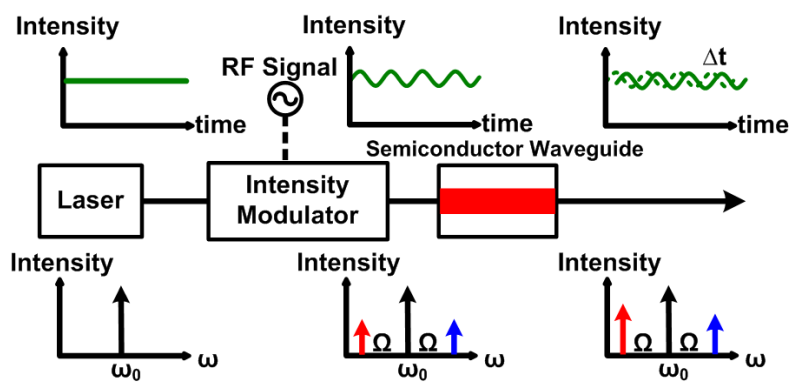

Fig. 2. Basic scheme of slow and fast light in semiconductor waveguides. The top and bottom rows are the time-domain and frequency-domain descriptions, respectively. $\omega 0$ : angular frequency of the laser; $\Omega$ : $R F$ signal frequency.

The RF phase shift $\Delta \varphi_{R F}$ can be controlled either optically by the input optical power, or electrically by the injection electrical current for an SOA or voltage for an EA, as an example shown in Fig. 3. For an SOA, $g_{0}>0$, $\Delta \varphi$ is negative, which means fast light. On the other hand, slow light dominates in an absorbing waveguide.

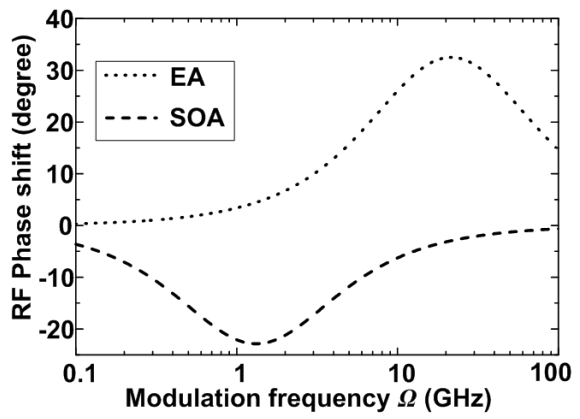

Fig. 3. Calculated RF phase shifts as a function of the modulation frequency. The phase shifts are induced by hanging the injection current for the SOA or bias voltage for the EA.

In [12], it is shown that for a double sideband input optical signal, the final RF phase shift is independent on the linewidth enhancement factor $\alpha$, which means that the refractive index dynamics does not influence the group velocity. Therefore the RF phase shift described is only governed by the gain/absorption dynamics. Reference [12] shows that the maximum phase shift is not enough to achieve the desired 360 degrees. To increase the phase shift a solution has been proposed.

We have recently proposed a method for exploiting the refractive index dynamics to enhance the RF phase shift [13]. In this case, after the propagation in the SOA one of the sidebands is blocked by the FBG filter. The experimental set-up is shown in Fig. 4. The network analyzer modulates the laser beam to generate two sidebands (red-shifted and blue-shifted). The modulated beam is coupled into a bulk SOA, where CPO and FWM effects will induce changes of the phase and the amplitude of the two sidebands. After the SOA, one of the 
two sidebands, red-shifted or blue-shifted, is blocked by the FBG notch filter before detection. The experimental controls are the input optical power to the SOA and the RF modulation frequency. By incorporating an optical fiber amplifier (EDFA) and a variable optical attenuator, the input optical power can be adjusted from $-10.3 \mathrm{dBm}$ to $13.6 \mathrm{dBm}$.

For three different optical filtering schemes, i.e., no optical filtering, blocking the red-shifted sideband and blocking the blue-shifted sideband, we have measured the phase and power change of the microwave modulation relative to its value at the minimum input optical power of $-10.3 \mathrm{dBm}$, as shown in Fig. 5 . The modulation frequency is $19 \mathrm{GHz}$. The results demonstrate that blocking the red-shifted sideband, shown by the blue curves in Fig. 5a, lead to positive phase changes and enhance the absolute RF phase shift from $\sim 15^{\circ}$ up to $\sim 150^{\circ}$, which corresponds to a ten-fold increase of the maximum phase shift obtained in the absence of filtering. When only the blue-shifted sideband and carrier are detected, the RF power in Fig. 5b shows a dip which is correlated with the sharp increase of the phase of the microwave signal. On the other hand, blocking the blue-shifted sideband only induces a small change in the RF phase shift and power, as the red curves show. The solid lines in Fig. 5 are numerical simulations based on an SOA model including carrier density depletion and FWM among the electrical field components and demonstrate very good agreement with the experimental results.

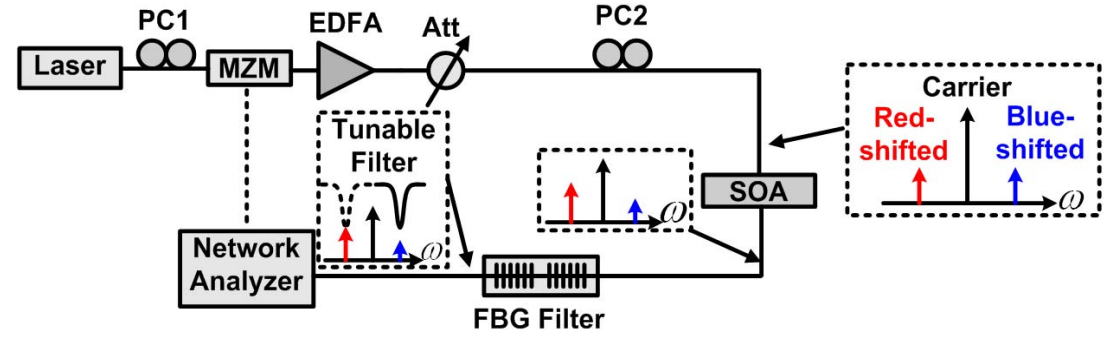

Fig. 4. Experimental set-up to enhance the light slow-down by optical filtering after the SOA.

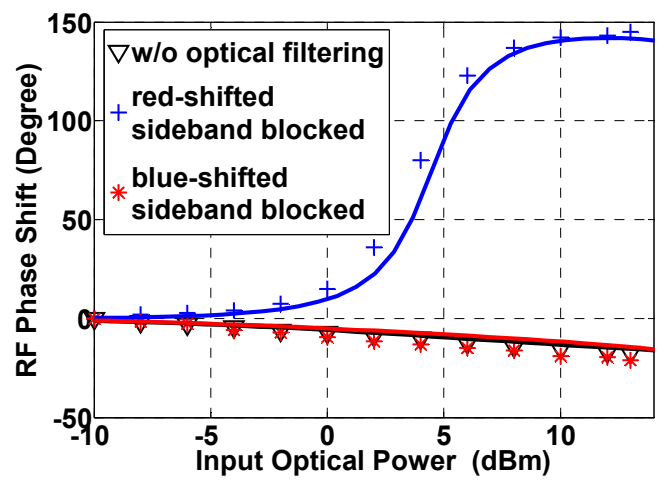

a)

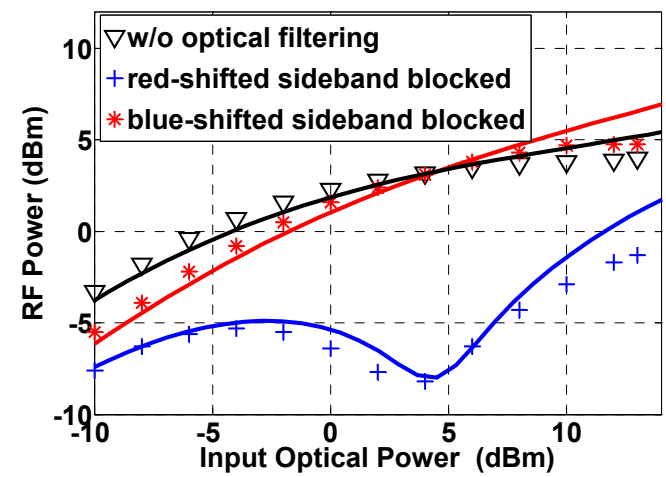

(b)

Fig. 5: (a) RF phase shifts and (b) RF power vs. the input optical power. The markers are experimental data taken at a modulation frequency of $19 \mathrm{GHz}$. The solid lines are simulation results.

\section{IMPLEMENTATIONS OF SFL EFFECT IN MICROWAVE PHOTONIC DEVICES}

To show the potential of SFL in semiconductor waveguides to implement a microwave phase shifter, we have implemented a tunable microwave photonic notch filter, which has the potential for applications in broadband wireless access networks and radar systems. This function can be realized by a microwave phase shifter capable of providing a tunable phase-shift over the 360 degrees. Recently, utilizing the SFL effect assisted by optical filtering and initial chirp effect, we have experimentally implemented a microwave photonic notch filter at around $30 \mathrm{GHz}$ with close to $100 \%$ fractional tuning range [14]. The schematic of the proposed microwave photonic notch filter is illustrated in Fig. 6. The filter itself is a simple Mach-Zehnder interferometer composed of two arms, one of which incorporates the microwave phase shifter, shown in the dotted-line box, which consists of an SOA followed by a FBG notch filter to block the red-shifted sideband. The EDFA is used to ensure that the SOA operates in the saturation regime. After the microwave phase shifter, a tunable attenuator provides amplitude balance between the two arms and does not influence the RF phase. The laser wavelength is $1552.7 \mathrm{~nm}$. The modulation frequency is sweeping between 29.75 and $30.25 \mathrm{GHz}$. The SOA current is tunable between 90 and $230 \mathrm{~mA}$, and is used to control the RF phase change. 


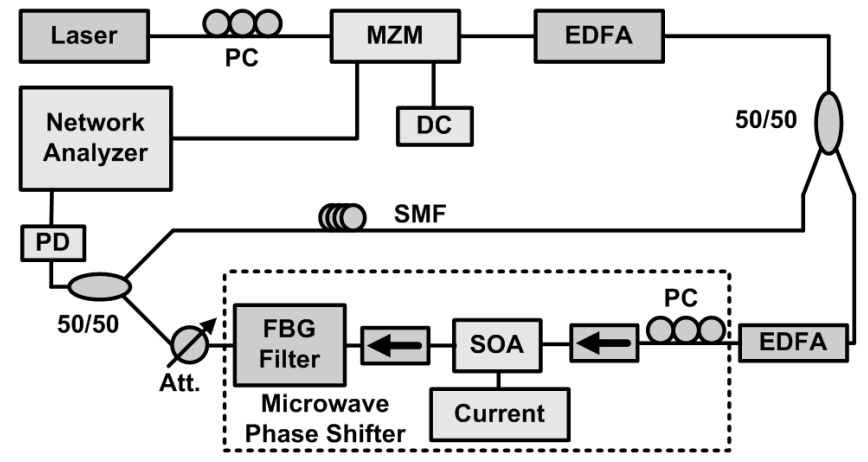

Fig. 6. Schematic diagram of a tunable two-tap microwave photonic notch filter.

The measured spectral responses of the proposed microwave photonic notch filter are shown in Fig. 7. The FSR is $9.4 \mathrm{MHz}$, corresponding to $22 \mathrm{~m}$ optical fiber length difference between the two arms, which is introduced by the EDFA. The result shows a $\sim 100 \%$ fractional tuning over a whole FSR by changing the injection current of the SOA and switching the operation points of the MZM. During the entire tuning range, the notch rejection is always larger than $30 \mathrm{~dB}$ and the shape of the spectral response is unchanged. Because the proposed microwave phase shifter based on the slow and fast light effects in an optical filtering assisted SOA can enhance the obtainable RF phase shift over several tens $\mathrm{GHz}$ bandwidth, this widely tunable demonstrated microwave photonic notch filter can also be applied at other microwave or millimeter-wave bands. And by decreasing the time difference between two arms, the $\sim 100 \%$ fractional tuning ability will maintain for even larger filter FSRs.

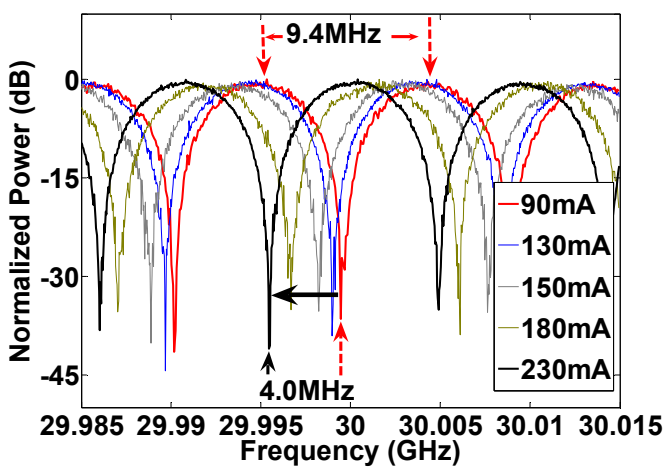

(a)

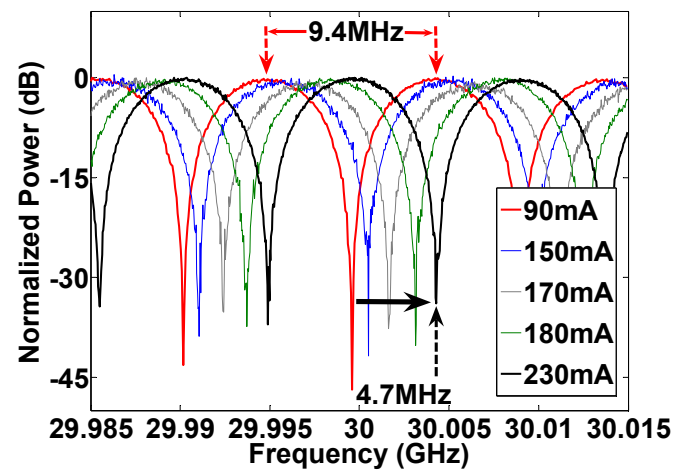

(b)

Fig. 7. RF spectral responses of the microwave photonic notch filter obtained by changing the injection current of the SOA, when the modulator is biased at (a) $\mathrm{V1}=4.5 \mathrm{~V}$ and (b) $\mathrm{V} 2=8.1 \mathrm{~V}$.

\section{CONCLUSIONS}

We have reviewed the basic theory of SFL effects due to the CPO effects in semiconductor waveguides. In order to further push the controllable RF phase shift to 360 degrees and the available bandwidth to higher frequency bands, we have introduce the use of an optical filter after the SOAs. We have also shown the feasibility of this PMPS in a microwave photonic filter application.

\section{ACKNOWLEDGEMENTS}

The authors would like to acknowledge the financial support from the Danish Research Councils through the QUEST project, the Spanish MICINN through Plan Nacional I+D TEC2007-68065-C03-01, as well as the European Union FP7 projects GOSPEL.

\section{REFERENCES}

[1] J. Capmany, and D. Novak, "Microwave photonics combines two worlds," Nature Photonics 1, 319-330 (2007).

[2] A. Seeds: Microwave photonics, IEEE Trans. Microwave Theory Tech. 50, 877-887 (2002).

[3] R.A. Minasian: Photonic signal processing of microwave signals, IEEE Trans. Microwave Theory Tech. 54, 832-846 (2006).

[4] K.P. Jackson, S.A. Newton, B. Moslehi, M. Tur, C.C. Cutler, J.W. Goodman, and H.J. Shaw: Optical fiber delay line signal processing, IEEE Trans. Microwave Theory and Techniques, MTT-33, 193-209 (1984).

[5] J.E. Heebner, V. Wong, A. Schweinsberg, R.W. Boyd and D.J. Jackson: Optical transmission characteristics of fiber ring resonators, IEEE J. Quant. Elect. 40, 726-730, (2004).

[6] N.A. Riza, IEEE/OSA J. Lightwave Technol. 12 (1994), 1440-1447. 
[7] J.L.Corral et al::True-time delay scheme for feeding optically controlled phased-array antennas using chirped-fiber gratings, IEEE Photon. Technol. Lett., vol. 9, no. , pp. 1529-1531, 1997.

[8] J. Han et al., IEEE/OSA J. Lightwave Technol., 21 (2003), 3257-3261.

[9] L. V. Hau, S. E. Harris, Z. Dutton, and C. H. Behroozi: Light speed reduction to 17 meters per second in an ultracold atomic gas, Nature 397, 594-598 (1999).

[10] M. S. Bigelow, N. N. Lepeshkin, and R. Boyd: Observation of ultraslow light propagation in a ruby crystal at room temperature, Phys. Rev. Lett. 90, 113903-1-4 (2003).

[11] P. Palinginis, M. Moewe, E. Kim, F. G. Sedgwick, S. Crankshaw, C. J. Chang-Hasnain, H. Wang, and S. L. Chuang: Ultra-slow light $(<200 \mathrm{~m} / \mathrm{s})$ in a semiconductor nanostructure, in Proc. CLEO, Post deadline paper CPDB6, Baltimore, USA, May 2005.

[12] A. Uskov, F. Sedgwick, and Chang-Hasnain: Delay limit of slow light in semiconductor optical amplifiers, IEEE Photon. Technol. Lett. 18, 731-733 (2006).

[13] W. Xue, Y. Chen, F. Öhman, S. Sales, and J. Mørk: Enhancing light slow-down in semiconductor optical amplifiers by optical filtering, Opt. Lett. 33, 1084-1086 (2008).

[14] W. Xue, S. Sales, J. Mørk, and J. Capmany: Widely tunable microwave photonic notch filter based on slow and fast light effects, IEEE Photon. Technol. Lett., vol. 21, no. 3, pp. 167-169, 2009. 